\title{
Avaliação dos conhecimentos oftalmológicos básicos em estudantes de Medicina da Universidade Federal do Piauí
}

\author{
Evaluation of basic ophthalmologic knowledge \\ in students of medicine Piaui federal university
}

João Batista Lopes Filho', Rubens Amorim Leite², Daniel Amorim Leite², André Rodrigues de Castro², Lorena Santana Andrade ${ }^{2}$

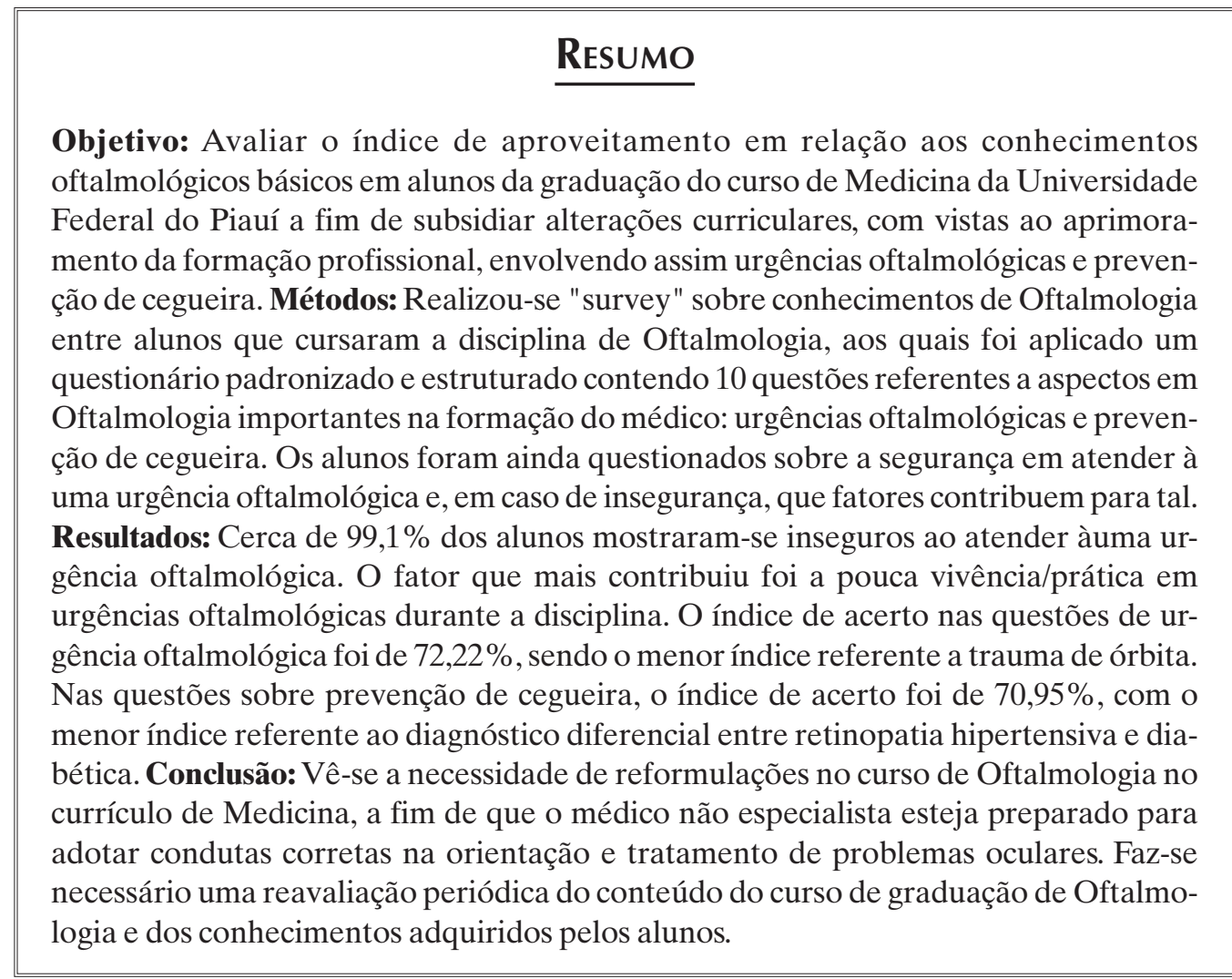

Descritores: Oftalmologia/educação; Educação médica; Avaliação; Currículo; Educação de graduação em Medicina

\footnotetext{
${ }^{1}$ Professor Doutor da disciplina de Oftalmologia da Universidade Federal do Piauí - UFPI - Teresina (PI), Brasil;

${ }^{2}$ Acadêmicos de Medicina da Universidade Federal do Piauí - UFPI - Teresina (PI), Brasil.

Universidade Federal do Piauí - UFPI - Teresina (PI), Brasil
}

Recebido para publicação em: 8/6/2010 - Aceito para publicação em 19/1/2011 


\section{INTRODUÇÃO}

A disciplina de Oftalmologia foi introduzida em caráter opcional no currículo médico brasileiro em 1885. Entretanto, a primeira reunião entre professores para discutir a importância da Oftalmologia na graduação somente aconteceu em 1981 na cidade de Campinas ${ }^{(1-2)}$.

As consultas oftalmológicas representam 9\% do atendimento médico global e 5\% das urgências médicas $^{(1-2)}$. Desse modo, é de fundamental importância que o médico generalista saiba fazer o manejo inicial de pacientes com queixas oftalmológicas. Até mesmo porque várias doenças cursam com algum grau de comprometimento oftalmológico, como hipertensão arterial sistêmica, diabetes melitus, colagenoses, etc ${ }^{(2)}$. Além disso, um médico não especialista é capaz de resolver $69 \%$ das urgências oftalmológicas e encaminhar corretamente $100 \%$ dos casos em que é necessário avaliação do oftalmologista ${ }^{(3)}$.

A literatura nacional é carente em informações sobre as características da qualidade do atendimento inicial oftalmológico ${ }^{(3)}$. Sabe-se, porém, que existem deficiências e que tal fato pode está associado a um despreparo durante o curso de graduação ${ }^{(1)}$.

Desse modo, aplicou-se um questionário com a proposta de avaliar o índice de aproveitamento em relação aos conhecimentos oftalmológicos básicos em alunos da graduação do curso de Medicina da Universidade Federal do Piauí, envolvendo urgências oftalmológicas e prevenção de cegueira.

\section{MéTOdos}

Realizou-se "survey" sobre conhecimentos de Oftalmologia entre alunos que cursaram a disciplina de Oftalmologia ( $\mathrm{n}=111)$ na Universidade Federal do Piauí (UFPI), aos quais foram aplicado um questionário padronizado e estruturado, contendo 10 questões referentes a aspectos em Oftalmologia importantes na formação do médico generalista: urgências oftalmológicas (seis questões) e prevenção de cegueira (quatro questões). Cada questão continha 05 alternativas, sendo apenas uma correta. Os alunos foram ainda questionados a respeito da segurança em atender uma urgência oftalmológica e se em caso de insegurança que fatores contribuem para tal. Nesta situação mais de uma opção poderia ser escolhida.

Foram incluídos no estudo alunos que cursaram a disciplina de Oftalmologia na UFPI, regularmente ma- triculados no $1^{\circ}$ período de 2006 . A disciplina é ministrada no sexto período da graduação, por quatro meses num total de 90 horas/aula. Questões com nenhuma ou mais de uma alternativa assinaladas foram excluídas.

\section{Resultados}

Apenas um estudante afirmou estar seguro em atender uma urgência oftalmológica. Cerca de 92,79\% dos estudantes afirmaram que tal insegurança decorre da pouca vivência ou prática em urgências (Figura 1). Houve uma média de $72,22 \%$ de acertos em todas as questões. O mesmo índice foi obtido nas questões referentes à urgência oftalmológica. Já na parte referente à prevenção de cegueira o índice foi de $71 \%$.

\section{Discussão}

De acordo com os dados deste estudo, vê-se que a preparação do aluno de graduação em Medicina não é a esperada. Sabe-se que este inquérito possui limitações, pelo fato da amostra envolver apenas alunos de uma única escola médica (Universidade Federal do PiauíUFPI), entretanto seus resultados são condizentes com outro estudo publicado há quase 10 anos $^{(2)}$. Nesse estudo concluiu-se que alunos do antigo $6^{\circ}$ ano das Faculdades de Medicina de São Paulo apresentavam importantes falhas em aspectos do conhecimento de Oftalmologia básicos para um médico generalista ${ }^{(2)}$.

Ao serem questionados sobre a segurança em atender uma urgência oftalmológica, 99,1\% dos alunos entrevistados da UFPI mostraram-se inseguros. Tal fato foi justificado pela maioria dos estudantes como sendo conseqüência da pouca vivência em urgências

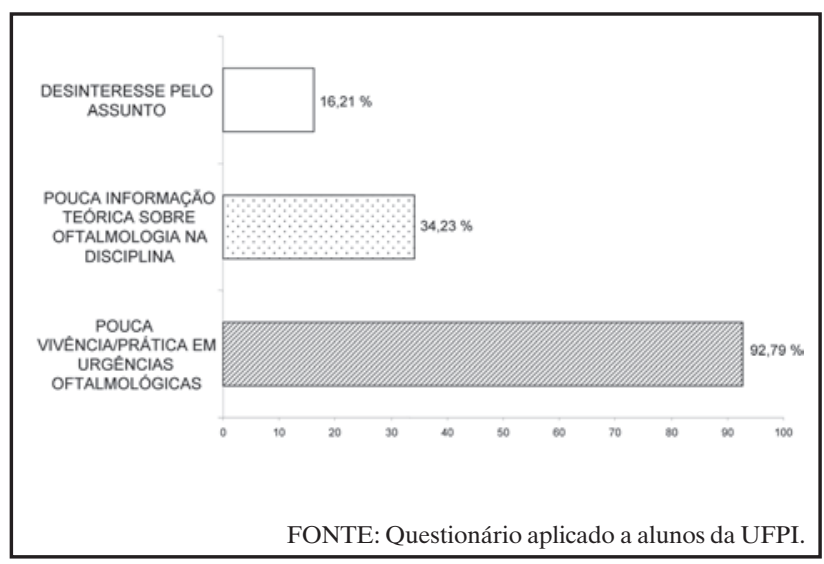

Figura 1 - Motivo da insegurança ao atender uma urgência oftalmológica entre alunos do curso de Medicina da UFPI. 


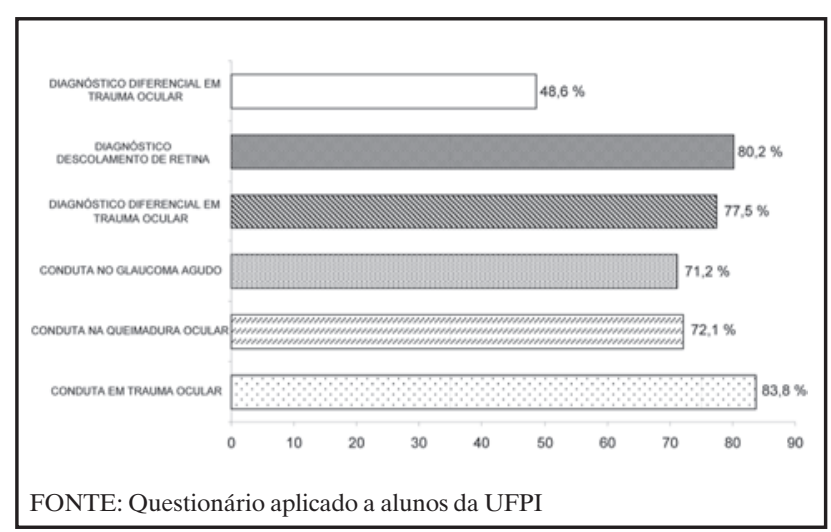

Figura 2 - Aproveitamento dos conhecimentos sobre urgências oftalmológicas entre os estudantes de Medicina da UFPI

oftalmológicas ${ }^{(3)}$. Isso demonstra a realidade de grande parte das escolas médicas brasileiras, onde há um grande número de alunos para um quadro cada vez mais reduzido de professores, o que acaba gerando sobrecarga e diminuição do aproveitamento durante as atividades práticas. Além disso, tais professores supervisionam os programas de residência médica, gerando mais sobrecarga $^{(4,5)}$.

Desse modo cria-se um ciclo vicioso, no qual o baixo aprendizado em atividades práticas acaba gerando desinteresse por parte dos alunos ${ }^{(3)}$. Isso talvez explique o resultado desse estudo, onde $16,21 \%$ dos alunos afirmaram que o motivo da sua insegurança em atender uma urgência oftalmológica seria por desinteresse pelo assunto.

De maneira geral, o índice de acerto no questionário aplicado foi de 71,8\%. Tal questionário era padronizado, de tal maneira que os alunos podem ter obtido um resultado acima do esperado. Isso ocorre porque tais graduandos podem ter respondido as questões através de eliminações. O ideal talvez seria aplicação de questionário não-padronizado, mas isso poderia criar um viés de subjetividade durante a correção deste. Ao elaborarse este questionário não era intenção confundir os estudantes, mas apenas avaliar o mínimo de conhecimento.

$\mathrm{O}$ índice de aproveitamento nas questões referentes ao atendimento de urgências oftalmológicas foi de $72,22 \%$, superior ao obtido nas questões relativas à prevenção de cegueira (71\%).

Na primeira questão foi obtido um índice de acerto de $83,8 \%$ (Figura 2). A hemorragia subconjuntival é uma situação comum no pronto-socorro e que o médico não-especialista poderá conduzir sem maiores dificuldades, bastando para isso um exame atento do sistema visual do paciente, com objetivo de afastar um quadro

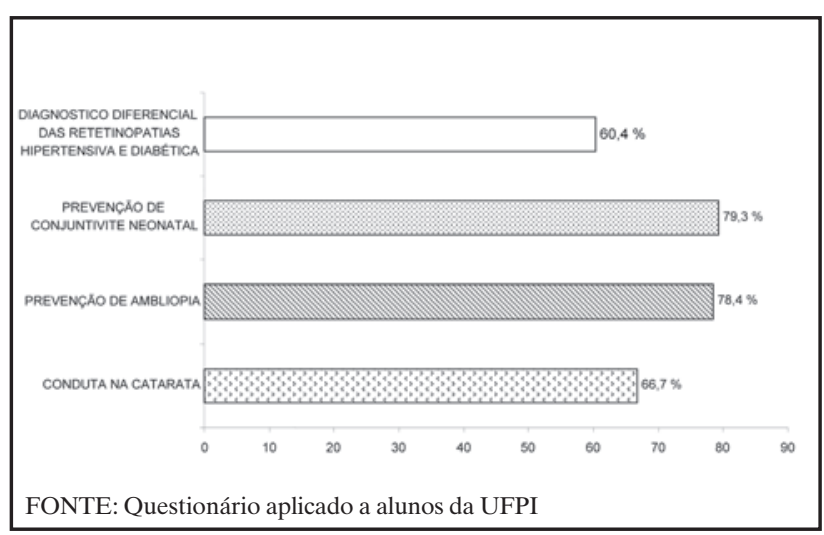

Figura 3 - Aproveitamento nas questões sobre prevenção de cegueira entre os estudantes de Medicina da UFPI.

de perfuração ocular. Assim o paciente será esclarecido sobre o caráter temporário do quadro, tranqüilizando$\mathrm{se}^{(3)}$.

Outro quadro comum em situações de urgência oftalmológica são as queimaduras oculares. Este assunto foi abordado na segunda questão do questionário, obtendo-se um acerto de 72,1\% (Figura 2). O manejo inicial correto é de fundamental importância para um bom prognóstico inicial destes pacientes. Assim sendo, devese lavar em abundância o olho acometido, não havendo indicação da utilização de substâncias que tentem neutralizar a substância envolvida no acidente. Em seguida, o paciente deverá ser então encaminhado para avaliação do oftalmologista ${ }^{(3,6)}$.

$\mathrm{Na}$ terceira questão foi abordado o atendimento inicial de um paciente com suspeita de glaucoma agudo. Tal diagnóstico pode ser suspeitado, associando-se alguns sintomas do quadro (vômitos e/ou dor ocular, por exemplo) com alguns sinais como pupila não reagente ou midríase média. Tais sinais podem ser explorados sem a necessidade de aparelhagem oftalmológica especializada, bastando para isso o uso de uma lanterna ${ }^{(2)}$. Observouse que $28,8 \%$ dos alunos não souberam diagnosticar tal situação, o que certamente irá acarretar prejuízo visual ao paciente, que não terá assim um atendimento inicial adequado (Figura 2). O diagnóstico e tratamento precoce do glaucoma são de fundamental importância para prevenção da perda visual, no entanto, dependem do conhecimento e da conduta dos médicos que fazem o primeiro atendimento $^{(6)}$. A utilização do manitol endovenoso por médico não-oftalmologista é medida salvadora em locais onde o acesso ao serviço especializado é demorado e algumas horas de espera podem ter como resultado a perda da visão por parte do paciente ${ }^{(3)}$.

Situações envolvendo suspeita de perfuração ou 
penetração ocular são também comuns em situações de urgência oftalmológica. Isso foi então abordado na quarta questão do questionário. Todo trauma ocular deve ser considerado como perfuração até prova contrária ${ }^{(3)}$. Mais uma vez, a associação entre história do mecanismo de trauma com um exame do sistema visual atento pode ser suficiente para suspeição do quadro e conduta acertada. Uma conduta intempestiva ou displicente pode acarretar em prejuízo da acuidade visual deste paciente ${ }^{(2,3)}$.

$\mathrm{Na}$ quinta questão temos um caso de descolamento de retina, que alerta para a importância da fundoscopia após trauma ocular contuso. Os sintomas do paciente devem servir como referência para o médico não oftalmologista solicitar um exame oftamológico completo, incluindo mapeamento de retina. Além do mais, durante esses traumas poderão ocorrer roturas retinianas, predisponentes ao descolamento de retina (necessitando de acompanhamento) ${ }^{(7)}$.

A última questão referente à urgência oftalmológica também abordou tema referente a trauma oftalmológico. A fratura do assoalho da órbita (fratura em blow-out) é o tipo mais frequente em trauma ocular. Desse modo, durante o primeiro atendimento destes pacientes o médico deve estar atento para esta possibilidade, até porque este evento pode acontecer em situações do cotidiano, como uma "bolada" na região orbitária, uma "cotovelada", acidente automobilístico etc. É uma situação preocupante na qual apenas $48,6 \%$ dos estudantes foram capazes de diagnosticar corretamente tal situação (Figura 2). Diploplia, enoftalmia e alterações na motilidade ocular podem ser a chave para o correto diagnóstico ${ }^{(3)}$.

Outra situação em que o médico generalista é importante é naquela referente a prevenção de cegueira. Na sétima questão é abordado o tema catarata, envolvendo a congênita, traumática e senil. Esta é a maior causa de cegueira no Brasil ${ }^{(2)}$. O índice de acerto nesta questão foi de apenas $66,7 \%$, de tal maneira que um percentual significativo apontou que os estudantes não têm segurança para afirmar que o único tratamento curativo para a catarata na atualidade é a facectomia com ou sem implante de lente intraocular ${ }^{(6)}$ (Figura 3). Tais médicos têm que possuir o mínimo de informação referente ao tema para que possam orientar os pacientes. No caso de catarata congênita a cirurgia faz-se necessário o mais cedo possível, já que nessas crianças o sistema visual estará desenvolvendo-se. Desse modo, evita-se a ocorrência de ambliopia (tema a ser abordado em seguida). A catarata traumática também está relacionada às urgências oculares (veja a importância do tema) e deve ter sempre seu diagnóstico suspeitado em situa- ções de trauma, principalmente com perfuração ou penetração oculares ${ }^{(2)}$

Ambliopia é outro tema importante durante o curso de graduação. Um médico que não possua informações corretas a respeito do desenvolvimento visual humano poderá não saber orientar em situações que envolvam este quadro. É o que acontece na oitava questão, onde uma mãe procura esclarecer-se sobre o possível problema visual do filho. Não que o médico necessariamente faça o diagnóstico etiológico do problema naquela situação, mas que saiba orientá-la corretamente para procurar auxílio oftalmológico o mais cedo possível, para evitar a ambliopia. Tal investigação não deve ser protelada! Neste estudo, $78,4 \%$ dos estudantes souberam esclarecer corretamente o quadro para a mãe (Figura 3)2,8.

A penúltima questão também é interessante, já que trata de temas que podem desencadear cegueira no neonato. Tem-se assim a conjuntivite gonocócica, a retinopatia da prematuridade, catarata congênita e tracoma como possíveis doenças que podem ocorrer. $\mathrm{O}$ uso do nitrato de prata é importante na prevenção da conjuntivite gonocócica. Seu uso não deve ser evitado, mesmo sabendo-se da possibilidade de conjuntivite química decorrente da ação do nitrato. A retinopatia da prematuridade também pode ser manejada de tal forma a evitar maiores danos sobre a retina do prematuro. Tais crianças expostas a determinados fatores de risco para retinopatia (prematuridade, baixo peso, oxigenioterapia, etc) devem ser rastreadas o mais rapidamente possível por oftalmologista experiente. Da mesma forma, outras infecções congênitas devem ser pesquisadas, já que podem afetar o sistema visual como a toxoplasmose, sífilis, clamídia, etc ${ }^{(2,8)}$.

A última questão faz referência a retinopatia hipertensiva e diabética. O índice de acerto foi de apenas $60,4 \%$, mostrando que os alunos desconhecem as alterações retinianas decorrentes da hipertensão arterial sistêmica e do diabetes melitus (Figura 3). Na hipertensão arterial sistêmica, a realização de fundoscopia é importante até mesmo para a tomada de condutas nas urgências hipertensivas ou mesmo no manejo ambulatorial de um paciente hipertenso. Com relação a retinopatia diabética o médico deverá informar ao paciente diabético sobre a necessidade de exames periódicos e a importância do controle da doença para evitar-se a progressão da perda visual ${ }^{(6)}$. Hoje, está muito difundida a idéia do mutirão do olho diabético, para onde então estes pacientes podem ser encaminhados para realização de exames regulares ou, se necessário, fotocoagulação ${ }^{(2)}$. 
Assim, pode-se observar como as questões abordadas fazem parte do cotidiano do médico não-especialista. Logicamente, não se pode extrapolar os resultados obtidos nesse estudo para a população total de estudantes de Medicina, pois os currículos dos cursos médicos são diferentes. Tal fato é mais marcante ainda com relação ao ensino de Oftalmologia na graduação, pois alguns cursos são ofertados em poucas semanas e outros em até quatro meses, como na UFPI. Gera-se assim uma diferença com relação à carga horária dos cursos ofere$\operatorname{cidos}^{(5)}$. O ideal seria então a elaboração de um programa central que contemplasse as diversas áreas da Oftalmologia de interesse do médico generalista. Outros estudos, semelhantes a este, devem ser incentivados para que possamos ter conhecimento da real situação dos cursos de Oftalmologia nas universidades.

\section{Conclusão}

Os alunos da UFPI obtiveram resultados satisfatórios ao responder questões relacionadas ao atendimento inicial em urgências oftalmológicas, bem como sobre prevenção da cegueira. Apesar disso, deve-se pensar em uma reformulação da disciplina de Oftalmologia para que ocorra otimização do aprendizado, visando a melhora do atendimento inicial referente a quadros oftalmológicos.

\section{Abstract}

Purpose: To evaluate the Piaui Federal University graduating medical students' knowledge in basic ophthalmology, in order to promote curricular changes in this course. Methods: Students that had already passed the ophthalmology course were asked to answer a standard questionnaire containing 10 multiple choice questions about some ophthalmology topics considered important in the general Doctor's graduation, such as blinding prevention and ophthalmic emergencies. The students were also asked if they would feel secure and comfortable if they had to manage an ophthalmologic urgency, and if not, why was that so. Results: $99,10 \%$ of students affirmed that would not feel secure enough if they had to manage an ophthalmologic urgency. The most important fact that implied that, according to the students, was the lack of experience/life practice during the ophthalmology classes. The correct answers index in the questions concerning urgency in ophthalmology was $72,22 \%$, where only $48,60 \%$ of them knew how to identify a blow-out fracture. As for the Blindness prevention questions, the correct answer index was $71 \%$, where just $60,40 \%$ of the students could identify the characteristics fundoscopic changes seen in hypertensive retinopathy. Conclusion: The data shows that Piaui Federal University medical students do not feel capable to perform the first management in ophthalmologic urgencies. It's necessary, thus, to think about a curricular renewal in the ophthalmology course of this University, in order to improve its general doctors' graduation.

Keywords: Ophthalmology/education; Education, medical; Evaluation; Curriculum; Education, medical, undergraduate

\section{RefERÊNCIAS}

1. Kara José AC, Passos LB, Kara José FC, Kara José N. Ensino extracurricular em Oftalmologia: grupos de estudos / ligas de alunos de graduação. Rev Bras Educ Méd. 2007;31(2):166-72.

2. Ginguerra MA, Ungaro ABS, Villela FF, Kara-José AC, KaraJosé N. Aspectos do ensino de graduação em oftalmologia. Arq Bras Oftalmol. 1998;61(5):546-50.

3. Espindola RF, Teixeira FC, Yamakami IM, Silva HRF, Freitas JAH. Análise dos conhecimentos básicos sobre urgências oftalmológicas em plantonistas não-oftalmologistas. Ara Bras Oftalmol. 2006;69(1):11-5.

4. Bellan L. Ophthalmology undergraduate education in Canada. Can J Ophthalmol. 1998;33(1):3-7. Comment in: Can J Ophthalmol. 1998;33(1):1-2.

5. Marcondes AM. Avaliação discente de um curso de oftalmologia. Rev Bras Educ Méd. 2002;26(3):171-4.

6. Temporini ER, Kara-José N, Gondim EL, Dantas FJ. Conhecimentos sobre saúde ocular entre profissionais de um hospital universitário. Medicina (Ribeirão Preto). 2002;35(1):53-61.

7. Oliveira PRP, Nutels MJSC, Giongo F, Moretti GRF, Sakae TM. Trauma ocular contuso envolvendo o segmento posterior: qual a melhor conduta? ACM Arq Catarin Med. 2008;37(2):85-91.

8. Gondim EL, Rocha JCP, Braga FT, Temporini ER, Kara-José N. Conhecimento em saúde ocular na infância de profissionais de Hospital Universitário. Arq Bras Oftalmol. 1996;59(4):351.

\footnotetext{
Endereço para correspondência:

Rubens Amorim Leite

Rua Demerval Lobão, no 1245

Bairro de Fátima

CEP 64048-100 - Teresina- (PI), Brasil

e-mail: rubensamorim1@hotmail.com
} 